\title{
Analysis on the Spatio-Temporal Characteristics of Urban Expansion and the Complex Driving Mechanism: Taking the Pearl River Delta Urban Agglomeration as a Case
}

\author{
Luo Liu, ${ }^{1}$ Jianmei Liu, ${ }^{1}$ Zhenjie Liu $\mathbb{D}^{1},{ }^{2}$ Xuliang $X u,{ }^{3}$ and Binwu Wang ${ }^{4}$ \\ ${ }^{1}$ Guangdong Province Key Laboratory for Land Use and Consolidation, South China Agricultural University, \\ Guangzhou 510642, China \\ ${ }^{2}$ Guangdong Provincial Key Laboratory of Urbanization and Geo-Simulation, School of Geography and Planning, \\ Sun Yat-sen University, Guangzhou 510275, China \\ ${ }^{3}$ State Key Laboratory of Resources and Environmental Information Systems, \\ Institute of Geographical Sciences and Natural Resources Research, Chinese Academy of Sciences, Beijing 100101, China \\ ${ }^{4}$ Policy Research Center, Ministry of Housing and Urban-Rural Development of the People's Republic of China, \\ Beijing 100835, China \\ Correspondence should be addressed to Zhenjie Liu; liuzhj66@mail2.sysu.edu.cn
}

Received 15 May 2020; Revised 8 August 2020; Accepted 8 September 2020; Published 15 September 2020

Academic Editor: Wen-Ze Yue

Copyright @ 2020 Luo Liu et al. This is an open access article distributed under the Creative Commons Attribution License, which permits unrestricted use, distribution, and reproduction in any medium, provided the original work is properly cited.

\begin{abstract}
Rapid urbanization on a global scale leads to a number of issues such as arable land occupation and ecological degradation. In this context, analyzing the driving mechanism of urban land expansion in complex urban systems facilitates sustainable urban development. Most relevant studies focused on the role of each driving factor and less considered the interaction between different factors spatially and temporally. In order to provide a deep insight into the complex driving mechanism of urban expansion, this study began with an analysis of the spatio-temporal characteristics of urban expansion in the Pearl River Delta (PRD) urban agglomeration in different periods. Then, the driving mechanism of urban expansion intensity (UEI) and urban expansion landscape pattern were analyzed, based on the interaction of different driving factors over space and time. The result showed as follows. (1) During the period of 2000-2008, the overall UEI in the PRD was higher than that of the period of 2008-2015. From 2000 to 2015, the most important source of urban land expansion was farmland, followed by forest land. Meanwhile, the decline in the proportion of outlying expansion type indicated that the urban land has gradually become more compact. (2) From 2000 to 2015, the socio-economic factors had a greater effect on UEI than natural factors. And the interaction between the pairs of factors on UEI during 2000-2008 was significantly stronger than that during 2008-2015. (3) The driving factors of the urban landscape expansion index during the period from 2008 to 2015 were far more powerful than 2000-2008. And the interaction between the pairs of factors on urban landscape expansion index during 2000-2008 was significantly stronger than that during 2008-2015.
\end{abstract}

\section{Introduction}

Since the 21st century, global urbanization has been advancing at a high speed [1]. Urbanization has become an important feature of global changes and the main way for human activities to transform the natural environment and climate [2-6]. Urbanization is a complex spatial behavior, which is affected by factors including policies, population, economy, and natural factors, and presents different patterns in different regions [7-9]. It is worth noting that such land use/land cover change is usually irreversible. Therefore, the spatio-temporal evolution of urbanization has been one of the focuses of global change research.

Nowadays, many developing countries are undergoing a rapid urbanization process. The rapid economic development and increasing industrialization of these areas have also brought a growing number of urban residents. In this context, a series of problems have arisen, such as the 
occupation of scarce farmland resources, traffic congestion, and ecological degradation, which seriously affect the effective functioning of urban systems [10-13]. Therefore, it is of practical significance for predicting the development trend of urban land use and guiding the sustainable development of cities to find out the existing problems and evolution rules in the urban complex system.

As an important measure of the urbanization process and a direct expression of urban land use, urban expansion has been widely used in the research of urbanization driving factors and mechanism [14, 15]. Previous studies have shown that urban expansion is driven by various factors including natural factors, socio-economic factors, and political factors [16-18]. In addition, the driving factors on urban expansion usually change spatially and temporally. For example, Li et al. investigated the driving factors across different regions in China in different periods [19]. The results showed that spatial scales are important to analyze the spatial heterogenity of driving factors and driving factors tended to be diverse over time. Therefore, it is necessary to consider the spatio-temporal change characteristics to explain the driving mechanism of urban expansion.

In order to reveal the relationship between different driving factors and urban expansion in detail, many studies have examined the effects of each factor on urban expansion. Specifically, existing studies on driving mechanism of urban expansion can be classified as qualitative and quantitative research based on the analysis method used. The qualitative research aims at analyzing the driving mechanism of urban expansion from the perspective of policies (such as the transformation of government functions) [20], economy (such as economic globalization) [21], population (such as migration) [22], and so on. For example, Wei examined the impacts of development policies and economic restructuring on China's urban expansion, and the results showed that urban expansion was the main response to the government's top-down policy change and economic transition [23]. Qualitative research can well explain the effects of factors on urban expansion, such as policies, which are difficult to quantify. However, the qualitative research is difficult to compare the differences among factors from different aspects and further analyze the spatio-temporal changes of driving mechanism. In addition, it is also easily affected by human subjective factors and background knowledge.

In comparison, quantitative research analyzes the driving mechanism of urban expansion by quantifying the relationship between urban expansion indices and various driving factors. Common methods used in quantitative research include logistic regression (LR) [24, 25] bivariate regression (BR) [26], multiple linear regression (MLR) [27], principal component analysis (PCA) [28], and analytic hierarchy process (AHP) [29]. For example, Seto et al. used a multivariate regression to analyze the main driving factors of global urban expansion from 1970-2000. The results showed that annual growth in GDP per capita was the main driving factor in China, while urban expansion in India and Africa was more driven by urban population growth, and GDP growth and population growth were the main driving factors in Europe and North America, respectively [30]. On the one hand, most quantitative methods can visualize the importance of different driving factors in numerical terms. On the other hand, some quantitative methods can dynamically explore the driving mechanism of urban expansion over time (such as BR and MLR), while some quantitative methods can reveal spatial heterogenity of driving mechanism (such as LR).

The common quantitative methods focus on the role of each driving factor and are unable to analyze how different driving factors interact with each other on urban expansion. In fact, the interaction of different driving factors has also been shown to have an important impact on the complex urban system [31]. In addition, the spatio-temporal change analysis is necessary to study the driving mechanism of urban expansion as mentioned earlier. However, few studies use quantitative methods to explore the interaction of different factors on urban expansion spatially and temporally.

Taking the Pearl River Delta (PRD) urban agglomeration as a case study, this research aims at exploring the interaction of driving factors spatially and temporally in order to reveal the complex driving mechanism of urban expansion from the following three aspects. (1) Reveal the evolution of the driving mechanism of urban expansion in the space and time dimension. (2) Simultaneously consider the impact of driving factors on urban expansion intensity and urban expansion landscape pattern. (3) Relative importance of driving factors, spatial heterogeneity of risk areas, and the interaction between factor pairs are analyzed in a quantitative fashion. Specifically, this study first analyzes the urban expansion intensity, sources of urban expansion land, and landscape patterns in PRD based on the land use change database obtained from Landsat and China-Brazil Earth Resource Satellite 1 (CBERS-1) images in 2000, 2008, and 2015. Then, we quantitatively compare the driving mechanism of urban expansion intensity and urban expansion landscape pattern in PRD spatially and temporally, thus providing valuable scientific information for future urban planning and sustainable use of land resources.

\section{Study Area and Data Sources}

2.1. Study Area. The Pearl River Delta (PRD) urban agglomeration is located in the south-central part of Guangdong Province with an area of $54,700 \mathrm{~km}^{2}$. It has a humid monsoon climate in the southern subtropics with sufficient water and heat conditions. PRD administratively includes nine prefecture-level cities of Guangzhou, Shenzhen, Zhuhai, Foshan, Huizhou, Zhaoqing, Jiangmen, Dongguan, and Zhongshan (Figure 1). In 2015, the resident population of PRD was 58.742 million, of which the proportion of urban population was $84.59 \%$. And the GDP in PRD reached 6,227 billion yuan. The PRD is one of the regions with the highest level of socio-economic and urbanization development in China. Together with Beijing-Tianjin-Hebei Urban Agglomeration and Yangtze River Delta Urban Agglomeration, they form China's three major regional economic centers [32]. Meanwhile, with the integration of Guangdong, Hong Kong, and Macao regional cooperation and development, PRD presents a multicenter, high-intensity, and aggregated 


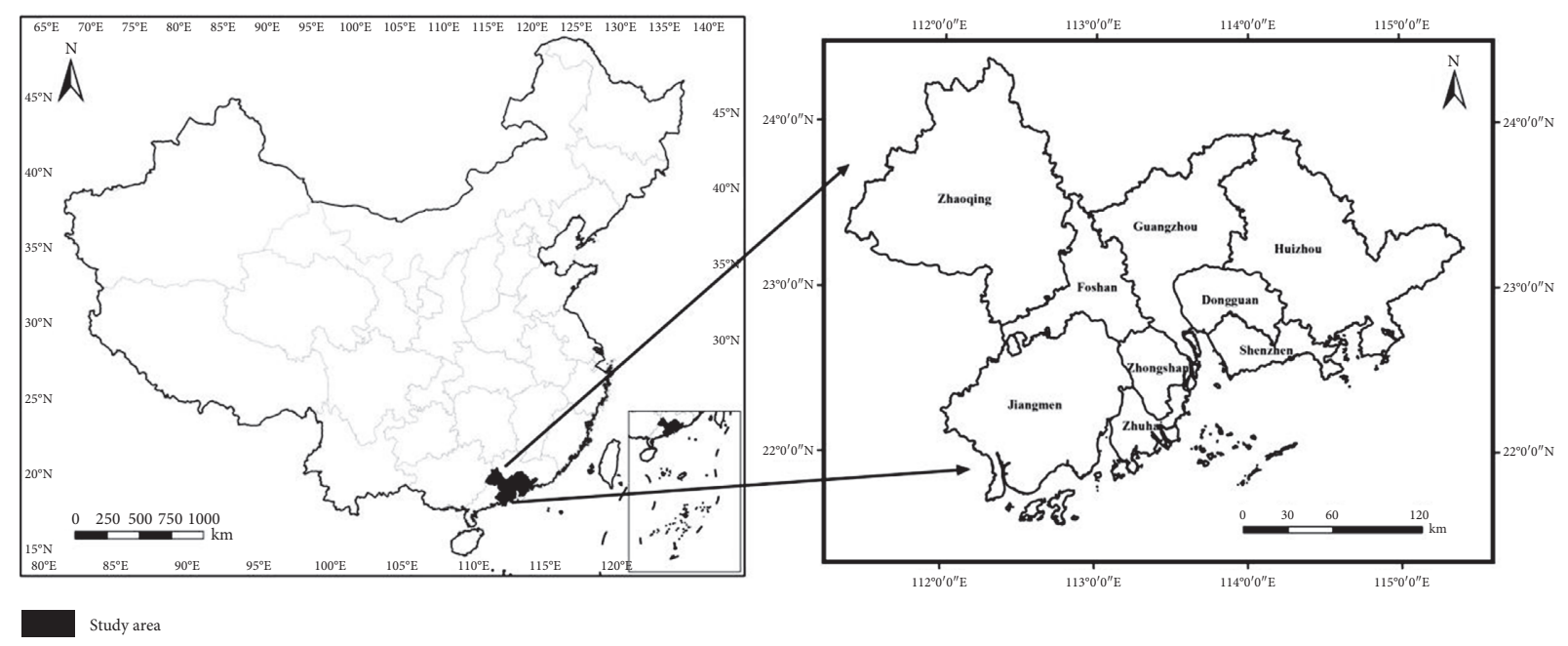

Figure 1: Study area and its location in China.

development mode. Therefore, studying the spatial-temporal evolution and driving mechanism of PRD is conducive to guiding the scientific planning of emerging urban clusters.

2.2. Data Sources. The land use database of the PRD in 2000, 2008, and 2015 is derived from the Resource and Environment Data Center of the Chinese Academy of Sciences, which extracts land use and land use change based on remote sensing satellite data including Landsat TM/ETM and CBERS-1 [33]. Specifically, the land classification system adopted divides the land use types into 6 primary types (farmland, forest land, grassland, water, urban and rural construction land, and unused land) and 25 secondary types. The evaluation accuracy of the primary types and secondary types are $94.3 \%$ and $91.2 \%$, respectively, which meets the research accuracy requirements for monitoring the urban expansion [34,35]. The sources of socio-economic statistics are China's urban statistical yearbooks and local statistical yearbooks in 2001, 2009, and 2016.

\section{Methodology}

This section introduces the theoretical framework of this study. As can be seen in Figure 2, we first extract urban expansion land from land use database in different periods. Then, urban expansion index, sources of urban expansion land, and landscape expansion index are adopted to analyze spatio-temporal characteristics of urban expansion. Finally, combined with natural and socio-economic factors, we explore the driving mechanism of urban expansion spatially and temporally.

3.1. Urban Expansion Intensity (UEI). UEI can be used to describe and analyze the speed and trend of different urban land expansions:

$$
I_{u e}=\frac{\Delta U_{i j}}{\Delta t_{j} \times \mathrm{TLA}_{i}} \times 100 \%,
$$

where $I_{u e}$ is the UEI, $\Delta U_{i j}$ is the urban expansion area of city $i$ in period $j, \Delta t_{j}$ is the time interval, and TLA $_{i}$ is the total land area of city $i$.

3.2. Sources of Urban Expansion Land. The process of urban expansion necessarily leads to encroachment on other land types, and the process is irreversible. The main sources of urban expansion land in different regions can be reflected through the proportion of other land types converting into urban land:

$$
P_{i j}=\frac{A_{i j}}{A_{i}} \times 100,
$$

where $P_{i j}$ is the proportion of the land use type $j$ being converted to urban land in city $i ; A_{i j}$ is the area of land use type $j$ occupied by urban expansion in city $i$; and $A_{i}$ is the total area of urban expansion in city $i$.

3.3. Landscape Expansion Index (LEI). The LEI breaks through the limitations of traditional landscape pattern index for landscape pattern analysis within a single point in time and can quantitatively reflect the dynamic changes of the landscape pattern over time [36]:

$$
\text { LEI }=\frac{A_{o}}{A_{o}+A_{v}} \times 100,
$$

where LEI is the landscape expansion index of the new patch, $A_{o}$ is the area where the new patch buffer intersects the original patch, and $A_{v}$ is the area where the new patch buffer intersects the blank area. The LEI range is $[0,100]$. When the LEI is $0,(0,50),[50,100]$, the corresponding landscape expansion patterns of the new spots belong to outlying type, edge-expansion type, and infilling type, respectively:

$$
\text { AWMEI }=\sum_{i=1}^{N} \operatorname{LEI}_{i} \times\left(\frac{a_{i}}{A}\right),
$$




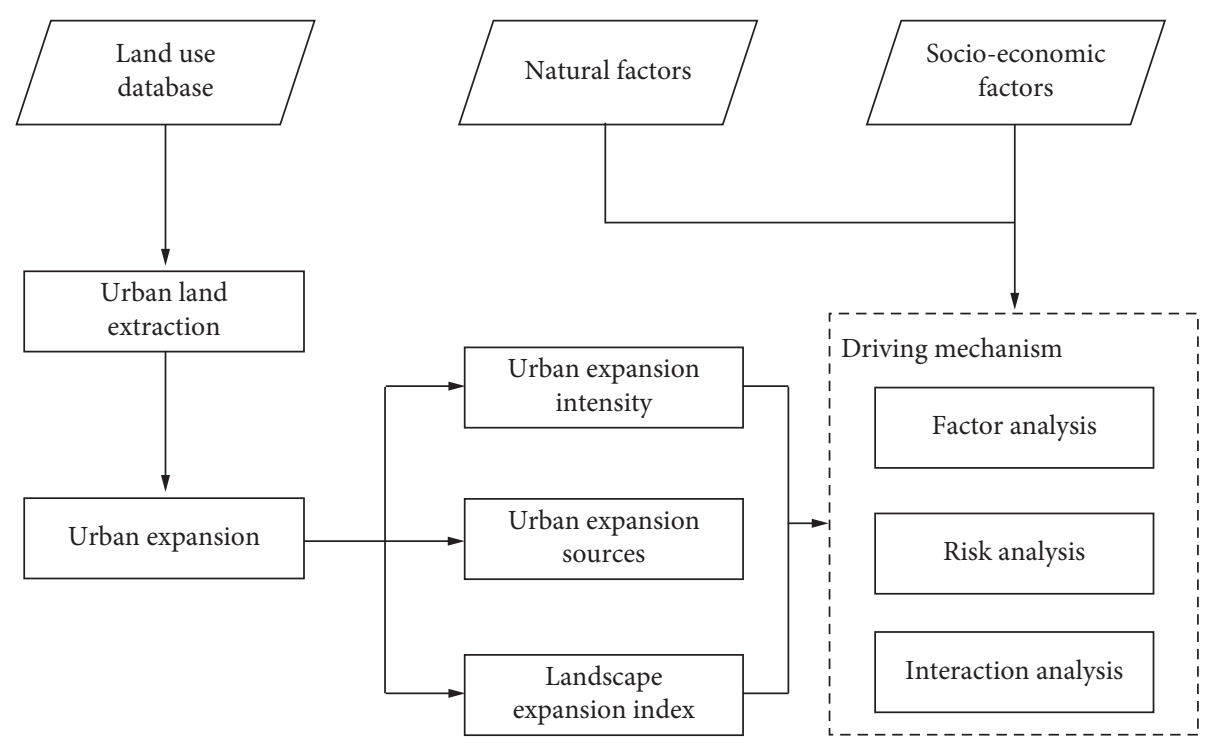

FIGURE 2: A schematic overview of research framework.

where AWMEI is the area-weighted mean LEI; $\mathrm{LEI}_{i}$ represents the LEI of the new patch $i ; a_{i}$ is the area of the new patch $i$; and $A$ is the total area of the new patches.

3.4. Driving Mechanism of Urban Expansion. In this study, we used the quantitative analysis method [37] to reveal the difference of the dominant driving factors of PRD in different periods and how the different driving factors interact with each other on the urban expansion. According to existing research on driving mechanism of urban expansion, the adopted driving factors include natural environmental factors and socio-economic factors [15, 31, 38-40]. Among them, digital elevation model (DEM), slope (Slope), distance to river (DTR), and distance to coastline (DTC) are used to characterize natural conditions; distance to railway and highway (DRH), population growth rate (PGR), Gross domestic product growth rate (GDPG), per capita wage growth rate $(\mathrm{PCWG})$, fixed asset investment growth rate (FAIG), and foreign investment growth rate (FIGR) are used to characterize socio-economic conditions.

3.4.1. Factor Analysis. The calculation of $P D$ values reflects the degree of consistency between urban expansion and the spatial distribution of driving factors, thus quantifying the relative importance of different driving factors:

$$
P D=1-\frac{1}{n \sigma^{2}} \sum_{i=1}^{N} n_{D, i} \sigma_{D, i}^{2}
$$

where $D$ is a driving factor; $n$ is the number of grids in which the study area is divided; $N$ is the number of subregions in which the driver factor is divided; $n_{D, i}$ is the number of grids of the factor in the subregion $i$, and $\sigma_{D, i}^{2}$ is the variance of the factor in the subregion $i$. When the $P D$ value is 0 , factor $D$ is completely irrelevant to urban expansion; when the $P D$ value is closer to 1 , the more factor $D$ determines urban expansion.
3.4.2. Risk Analysis. The risk areas of the factors were analyzed by using a $t$-test to compare the differences between the mean values of factor $D$ in different subregions. In this study, the average of UEI and LEI are calculated for each subregion:

$$
I=\frac{1}{n_{D, i}} \sum_{1}^{n_{D, i}} y_{D, i},
$$

where $I$ indicates the average risk value; $n_{D, i}$ is the number of grids of factor $D$ in the subregion $i$; and $y_{D, i}$ is the UEI or LEI of factor $D$ for each grid in the subregion $i$. The higher the $I$ value, the higher the UEI or LEI. Risk analysis makes it easy to compare the impact of the same factor at different levels.

3.4.3. Interaction Analysis. Interaction analysis identifies whether different driving factors interact independently of each other, or enhance or weaken each other. The five specific types of interaction are as follows:

(1) Nonlinear weaken: $P D\left(x_{1} \cap x_{2}\right)<\operatorname{Min}\left(P D\left(x_{1}\right)\right.$, $\left.P D\left(x_{2}\right)\right)$

(2) Uni-enhance/weaken: $\operatorname{Min}\left(P D\left(x_{1}\right), P D \quad\left(x_{2}\right)\right)$ $<P D\left(x_{1} \cap x_{2}\right)<\operatorname{Max}\left(P D\left(x_{1}\right), P D\left(x_{2}\right)\right)$

(3) Bienhance: $\operatorname{Max}\left(P D\left(x_{1}\right), P D\left(x_{2}\right)\right)<P D\left(x_{1} \cap x_{2}\right)$ $<P D\left(x_{1}\right)+P D\left(x_{2}\right)$

(4) Independent: $P D\left(x_{1} \cap x_{2}\right)=P D\left(x_{1}\right)+P D\left(x_{2}\right)$

(5) Nonlinear enhance: $P D\left(x_{1} \cap x_{2}\right)>P D\left(x_{1}\right)+P D$ $\left(x_{2}\right)$

\section{Results}

4.1. Urban Expansion Intensity. It can be seen from Figure 3 that the UEI of PRD was 0.74\% during 2000-2008, higher than $0.19 \%$ during 2008-2015. And except Jiangmen and Zhaoqing, each city also showed a stronger UEI during 2000 to 2008. In particular, UEI of Dongguan was the highest in 


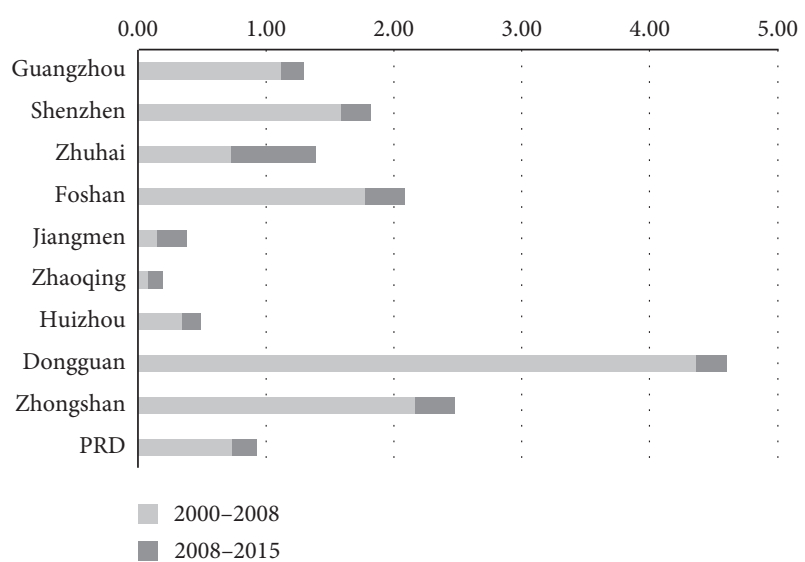

Figure 3: Urban expansion intensity of the Pearl River Delta urban agglomeration during 2000-2008 and 2008-2015.

PRD in the former period, reaching $4.37 \%$, while it was only $0.23 \%$ in the latter period, with a difference of about 19 times. The order of UEI during 2000-2008 was Dongguan $>$ Zhongshan $>$ Foshan $>$ Shenzhen $>$ Guangzhou $>$ Zhuhai $>$ Huizhou > Jiangmen > Zhaoqing; the order of LEI during 2008-2015 was Zhuhai $>$ Foshan $>$ Zhongshan $>$ Dongguan $>$ Shenzhen $>$ Jiangmen $>$ Guangzhou $>$ Huizhou $>$ Zhaoqing.

It can be also found that the UEIs in the six cities with relatively developed economies surrounding the Pearl River estuary were significantly higher than that in Jiangmen, Zhaoqing, and Huizhou, which are located in the peripheral areas. During 2000-2008, although Guangzhou, Shenzhen, and Zhuhai were the three most economically developed cities in PRD, their UEIs were lower than in Dongguan, Zhongshan, and Foshan. This was because since the reform and opening up, due to the support of national policies and the advantages of their geographical location, they have gone through the process of high-speed economic development, large population accumulation, and continuous urban expansion, and a large amount of land suitable for development and construction has been occupied. Dongguan, Zhongshan, and Foshan have continuously improved their investment environment, promoting the development of local characteristic economy, private economy, and exportoriented economy. The urbanization process was developing rapidly, of which Dongguan is the most typical city. During 2008-2015, Zhuhai had the highest UEI, reaching $0.66 \%$. This is mainly due to the implementation of "Hengqin Overall Development Plan" after 2008, which has gradually made Zhuhai Hengqin Free Trade Zone a demonstration region for a new mode of cooperation between Guangdong, Hong Kong, and Macao and has expanded the demand for urban expansion.

4.2. Spatial Distribution of Urban Expansion. The urban expansion of PRD showed significantly different spatial distribution characteristics in different periods (Figure 4). During 2000-2008, the urban land has undergone dramatic large-scale expansion centered on the Pearl River estuary. The urban expansion is mainly concentrated in Shenzhen,
Foshan, Dongguan, Zhongshan, and southwest of Guangzhou. And as the distance increases, urban land gradually becomes sporadic. During 2008-2015, urban land was evenly distributed within PRD, and there was no significant expansion compared with the previous period. This is mainly because, with the rapid development of the social economy of PRD and the continuous advancement of the industrialization, the land suitable for the development of urban construction land has continued to decrease. Meanwhile, with the continuous improvement of national and local governments' awareness of the protection of farmland resources and ecological environment, more attention has been paid to fully exploiting the potential of construction land, such as the "three old" transformation and the policy of "linking the increase and decrease of urban and rural construction land". Therefore, the UEI in PRD in the latter period is weaker than that in the former period.

4.3. Land Sources of Urban Expansion. The urban expansion area of PRD during 2000-2008 was $2709.34 \mathrm{~km}^{2}$. And the main land source was cultivated land, accounting for $64.36 \%$ of the total urban expansion area, followed by forest land and water (Table 1). Dongguan, Guangzhou, and Foshan had the larger urban expansion areas, which together accounted for $61.28 \%$ of the total urban expansion area of PRD. Among them, the main land sources for urban expansion in Dongguan and Guangzhou were farmland and forest land, while those in Foshan were farmland and water. The urban expansion in Zhuhai and Zhaoqing occupied relatively few other land types, only $77.00 \mathrm{~km}^{2}$ and $91.08 \mathrm{~km}^{2}$, respectively.

The urban expansion of PRD during 2008-2015 was $498.54 \mathrm{~km}^{2}$, only $18.40 \%$ of the previous period (Table 2 ). The main land sources for urban expansion were farmland and forest land, which accounted for $49.68 \%$ and $31.54 \%$ of the total area of urban expansion, respectively. Although the urban expansion areas in different cities has declined, those in Foshan and Guangzhou were still relatively large, accounting for $35.54 \%$ of the total area of urban expansion in PRD. And the main land sources for urban expansion in Foshan and Guangzhou were farmland, which together account for $47.28 \%$ of the total occupied farmland area in PRD. The occupation of forest land and water were mainly concentrated in Jiangmen and Zhuhai, with an area of $33.61 \mathrm{~km}^{2}$ and $33.91 \mathrm{~km}^{2}$, respectively.

4.4. Urban Expansion Landscape Pattern. According to Table 3, compared with the previous period, AWMEI increased by $2.86 \%$ in the latter period. Specifically, the proportion of infilling-expansion type and edge-expansion type increased by $0.93 \%$ and $8.14 \%$, respectively. As the main mode of urban expansion in PRD, outlying-expansion type decreased from $76.00 \%$ to $66.93 \%$, which indicated that the urban expansion was gradually becoming compact.

The changes of AWMEI and urban expansion types in Guangzhou, Foshan, Huizhou, Dongguan, and Zhongshan were consistent with those in PRD. Among them, AWMEI in Dongguan and Zhongshan rose by $14.44 \%$ and $13.68 \%$, respectively. This was closely related to the sharp increase in 


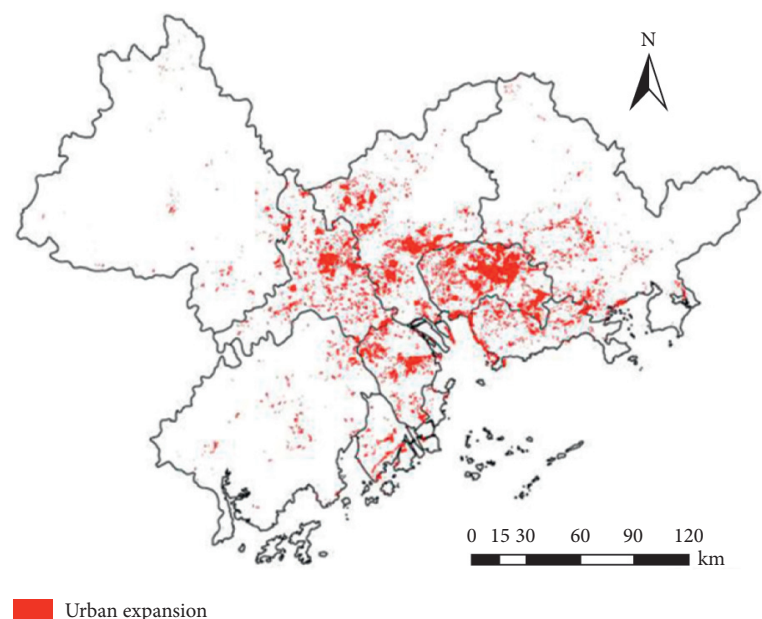

(a)

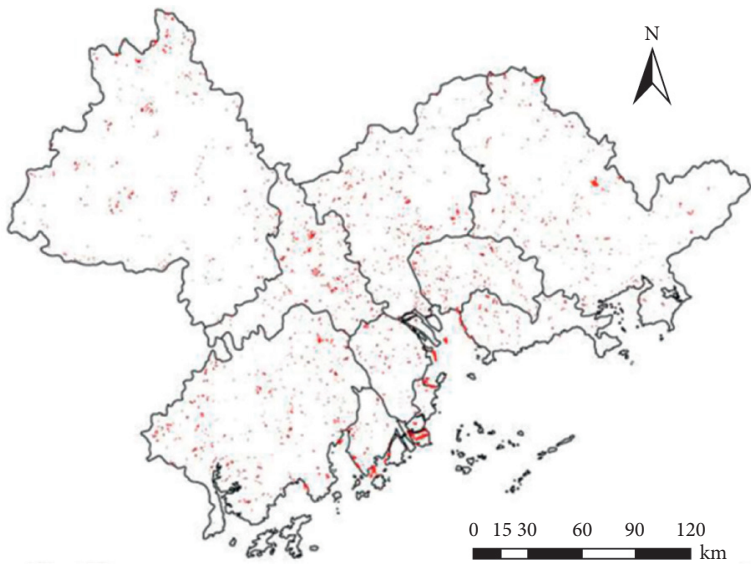

Urban expansion

(b)

Figure 4: Spatial expansion characteristics of the Pearl River Delta urban agglomeration during (a) 2000-2008 and (b) $2008-2015$.

TABle 1: Sources of urban expansion land in the Pearl River Delta urban agglomeration during 2000-2008 $\left(\mathrm{km}^{2}\right)$.

\begin{tabular}{|c|c|c|c|c|c|}
\hline Region & Farmland & Forest land & Grassland & Water & Sum \\
\hline Guangzhou & 400.42 & 121.67 & 0.65 & 23.11 & 545.85 \\
\hline Shenzhen & 114.42 & 70.24 & 0 & 29.65 & 214.31 \\
\hline Zhuhai & 51.67 & 12.55 & 0.75 & 12.04 & 77 \\
\hline Foshan & 297.03 & 49.77 & 0 & 153.3 & 500.1 \\
\hline Jiangmen & 44.87 & 55.06 & 0.92 & 22.72 & 123.56 \\
\hline Zhaoqing & 42.86 & 36.56 & 0 & 11.65 & 91.08 \\
\hline Huizhou & 214.24 & 73.34 & 1.12 & 10.03 & 298.73 \\
\hline Dongguan & 465.65 & 104.65 & 0 & 44.01 & 614.32 \\
\hline Zhongshan & 112.65 & 38.39 & 0 & 93.35 & 244.39 \\
\hline PRD & 1743.81 & 562.23 & 3.43 & 399.86 & 2709.34 \\
\hline
\end{tabular}

TABle 2: Sources of urban expansion land in the Pearl River Delta urban agglomeration during 2008-2015 $\left(\mathrm{km}^{2}\right)$.

\begin{tabular}{|c|c|c|c|c|c|c|}
\hline Region & Farmland & Forest land & Grassland & Water & Unused land & Sum \\
\hline Guangzhou & 56.81 & 23.11 & 2.64 & 4.94 & 0 & 87.5 \\
\hline Shenzhen & 8.36 & 14.96 & 0.72 & 2.25 & 0 & 26.28 \\
\hline Zhuhai & 11.67 & 4.5 & 1.17 & 33.91 & 0 & 51.25 \\
\hline Foshan & 60.29 & 15.56 & 0.24 & 13.6 & 0 & 89.69 \\
\hline Jiangmen & 39.73 & 33.61 & 2.29 & 7.5 & 0.36 & 83.48 \\
\hline Zhaoqing & 10.17 & 18 & 0.7 & 3.13 & 0 & 32 \\
\hline Huizhou & 25.26 & 25.38 & 1.76 & 1.96 & 0 & 54.35 \\
\hline Dongguan & 21.51 & 16.43 & 2.54 & 6.9 & 0 & 47.37 \\
\hline Zhongshan & 13.88 & 5.67 & 0 & 7.07 & 0 & 26.61 \\
\hline PRD & 247.67 & 157.21 & 12.05 & 81.24 & 0.36 & 498.54 \\
\hline
\end{tabular}

the proportion edge-expansion type and the sharp decrease in the proportion of outlying-expansion type. Although AWMEI in Shenzhen, Zhuhai, and Zhaoqing rose, the change was relatively small. It was worth noting that only AWMEI in Jiangmen showed a downward trend, with the proportion of infilling-expansion type and edge-expansion type falling by $2.95 \%$ and $2.37 \%$, respectively, while the proportion of outlying-expansion type rising to $81.88 \%$.

\section{Driving Mechanism of Urban Expansion in PRD during 2000-2015}

\subsection{Driving Mechanism of Urban Expansion Intensity}

5.1.1. Factor Analysis. PD values calculated through factor analysis can reflect the influence of potential driving factors on UEI, while risk analysis can reveal the average UEI of 
TABle 3: Changes of the urban landscape expansion index of the Pearl River Delta urban agglomeration during 2000-2008 and 2008-2015(\%).

\begin{tabular}{|c|c|c|c|c|c|c|c|}
\hline \multirow{2}{*}{ Region } & \multicolumn{3}{|c|}{ 2000-2008 } & \multicolumn{3}{|c|}{ 2008-2015 } & \multirow{2}{*}{$\triangle \mathrm{AWMEI}$} \\
\hline & Infilling & Edge-expansion & Outlying & Infilling & Edge-expansion & Outlying & \\
\hline Guangzhou & 4.96 & 31.45 & 63.59 & 7.36 & 38.52 & 54.12 & 5.18 \\
\hline Shenzhen & 2 & 53.72 & 44.28 & 6.09 & 43.67 & 50.24 & 1.94 \\
\hline Zhuhai & 5.67 & 30.34 & 63.99 & 2.61 & 47.79 & 49.6 & 0.49 \\
\hline Foshan & 5.52 & 16.78 & 77.7 & 6.2 & 49.91 & 43.9 & 4.95 \\
\hline Jiangmen & 5.68 & 17.76 & 76.56 & 2.73 & 15.39 & 81.88 & -2.25 \\
\hline Zhaoqing & 0.4 & 9.54 & 90.06 & 0.92 & 7.92 & 91.16 & 0.25 \\
\hline Huizhou & 0.17 & 14.84 & 84.99 & 2.34 & 15.85 & 81.81 & 2.76 \\
\hline Dongguan & 0.19 & 11.08 & 88.74 & 4.56 & 58.61 & 36.83 & 14.44 \\
\hline Zhongshan & 3.46 & 20.78 & 75.75 & 7.67 & 65.05 & 27.28 & 13.68 \\
\hline PRD & 2.87 & 21.14 & 76 & 3.8 & 29.28 & 66.93 & 2.86 \\
\hline
\end{tabular}

each driving factor in different risk areas. According to the results of factor analysis, the overall socio-economic factors showed greater influence on the UEI than natural factors, whether during 2000-2008 or 2008-2015. The specific results were as follows. During 2000-2008, GDPG (0.645) $>$ FAIG $\quad(0.461)>$ DRH $\quad(0.220)>$ DTC $\quad(0.216)>$ FIGR $(0.197)>$ PGR $\quad(0.154)>$ DEM $\quad(0.142)>$ PCWG $\quad(0.088)$ $>$ Slope (0.079) $>$ DTR (0.008). During 2008-2015, GDPG $(0.277)>$ FAIG $\quad(0.260)>$ DTC $\quad(0.209)>$ PCWG $\quad(0.153)$ $>$ DEM $\quad(0.090)>$ FIGR $\quad(0.089)>$ DRH $\quad(0.074)>$ PGR $(0.068)>$ Slope $(0.046)>$ DTR $(0.023)$. It can be found that GDPG and FAIG have always been the two factors that had the greatest impact on the UEI during 2000-2015. As for natural factors, DTR and Slope have relatively little influence due to the regional characteristics of sufficient water and heat, dense river network, and flat terrain. Only DTC had a relatively high $P D$ value, which was in the third place of all driving factors in both periods. Therefore, the following risk analysis was mainly conducted for GDPG, FAIG, and DTC.

5.1.2. Risk Analysis. According to Figure 5, it was easy to infer that whether in the previous period or the latter period, the closer the distance to the coastline, the stronger the urban expansion. On the one hand, as a frontier region of opening to the outside world, PRD had certain preferential policies. On the other hand, PRD was adjacent to Hong Kong and Macao and has convenient transportation, which made it easier to attract foreign investment and labor accumulation, thus promoting economic development and urbanization. Different from DTC, GDPG and FAIG showed different laws in the two periods. From 2000 to 2008, GDPG and FAIG in relatively developed cities surrounding the Pearl River estuary were relatively high. The rapid development of social economy and investment in fixed assets had a clustering effect on capital, population, and technology. At the same time, capital investment and the improvement of urban infrastructure were conducive to the inflow of labor and the scale development of secondary and tertiary industries. Therefore, the regional cities with larger GDPG and FAIG had relatively high UEI during 2000-2008. However, the regional cities with larger GDPG and FAIG had relatively low UEI during 2008-2015. This was mainly due to the relatively backward areas in PRD, such as Zhaoqing, Huizhou, and Jiangmen, where the regional GDP and investment in fixed assets were growing at a faster rate. The basic conditions for population attraction and urban development were not as good as those of relatively developed cities, so the demand for urban construction land was still lower than that of relatively developed cities.

5.1.3. Interaction Analysis. On the whole, the interaction between various factor pairs on the UEI during 2000-2008 was significantly stronger than that during 2008-2015 (Tables 4 and 5). During 2000-2008, the average PD value of socio-economic factor pairs $(0.66)>$ natural and socioeconomic factor pairs $(0.41)>$ natural factor pairs (0.19). During 2008-2015, the average $P D$ value of socio-economic factor pairs $(0.44)>$ natural and socio-economic factor pairs $(0.27)>$ natural factor pairs (0.17). The two periods showed common characteristics: the UEI was more driven by socioeconomic factor pairs than natural factor pairs, and the interaction of the any two factors was an enhancement.

The interaction between DEM and DTC was the strongest among the natural factor pairs, regardless of the previous period or the later period. This was mainly due to the convenient transportation conditions near the coastline in PRD, which was more suitable for the development of trade and foreign investment. The flat terrain weakened the restriction of natural environment on human activities and was suitable for urban expansion.

PRD is one of the frontier areas of national socio-economic development. The high GDP growth rate provided sufficient sources of capital for urban construction. Coupled with the rise in per capita wages, a large number of labor forces were gathered here. In order to meet the living and production needs of more nonagricultural population, the UEI was continuously strengthening. Therefore, the interaction between GDPG and PCWG was the strongest among the socio-economic factors in the two periods.

For natural and socio-economic factor pairs, DTC and GDPG had the strongest interaction during 2000-2008, while DTC and PCWG had the strongest interaction during 2008 and 2015. This was because the large-scale operation of the secondary and tertiary industries and the increase in 

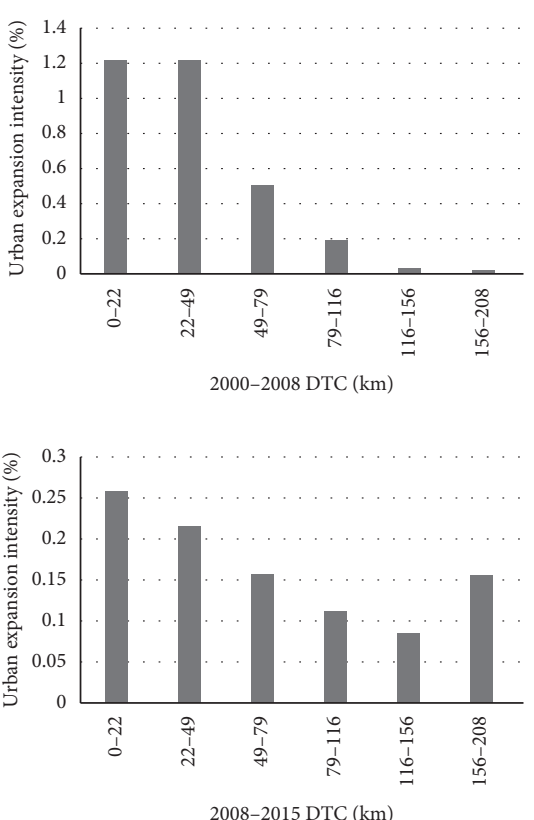
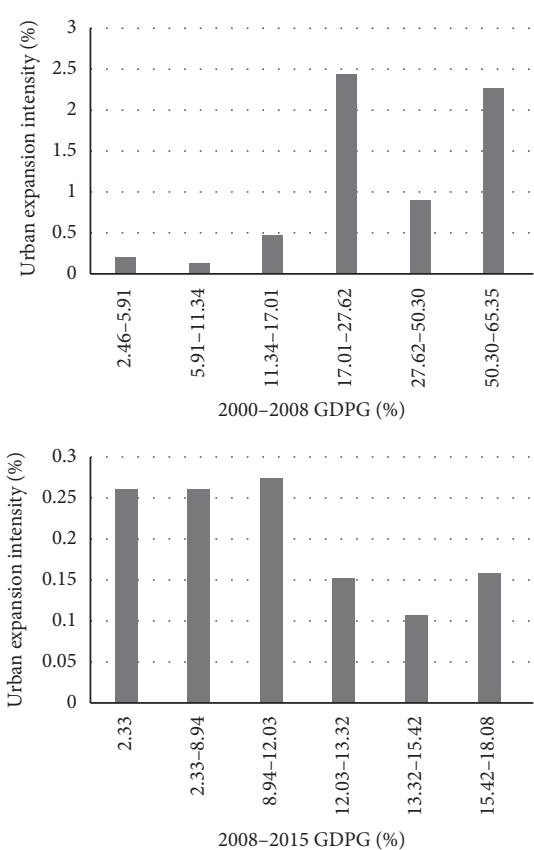
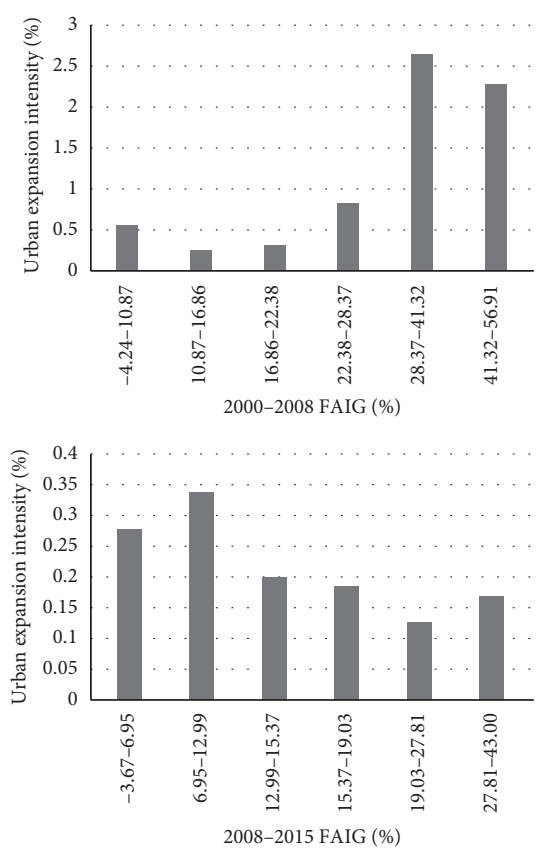

FIGURE 5: Risk analysis of DTC, GDPG, and FAIG on urban expansion intensity during 2000-2008 and 2008-2015.

TABLE 4: Interaction of different factor pairs on urban expansion intensity during 2000-2008.

\begin{tabular}{|c|c|c|c|c|c|c|c|c|c|c|c|}
\hline \multirow{2}{*}{ Driving factors } & & \multicolumn{4}{|c|}{ Natural factors } & \multicolumn{6}{|c|}{ Socio-economic factors } \\
\hline & & DEM & Slope & DTR & DTC & $\mathrm{DRH}$ & PRG & GDPG & PCWG & FAIG & FIGR \\
\hline \multirow{4}{*}{ Natural factors } & DEM & & & & & & & & & & \\
\hline & Slope & 0.15 & & & & & & & & & \\
\hline & DTR & $0.16^{\nearrow}$ & $0.09^{\nearrow}$ & & & & & & & & \\
\hline & DTC & $0.26^{*}$ & 0.25 & $0.25^{\nearrow}$ & & & & & & & \\
\hline \multirow{6}{*}{ Socio-economic factors } & DRH & 0.25 & 0.24 & $0.24^{\nearrow}$ & 0.33 & & & & & & \\
\hline & PRG & $0.37^{\nearrow}$ & $0.26^{\nearrow}$ & $0.19^{\nearrow}$ & $0.38^{\nearrow}$ & $0.41^{\nearrow}$ & & & & & \\
\hline & GDPG & 0.66 & 0.66 & $0.66^{\nearrow}$ & $0.74^{*}$ & 0.69 & $0.83^{\nearrow}$ & & & & \\
\hline & PCWG & $0.32^{\nearrow}$ & $0.21^{\nearrow}$ & $0.13^{\nearrow}$ & $0.59^{\nearrow}$ & $0.36^{\nearrow}$ & $0.61^{\nearrow}$ & $0.92^{\nearrow *}$ & & & \\
\hline & FAIG & 0.52 & 0.49 & $0.50^{\nearrow}$ & 0.67 & 0.55 & $0.77^{\nearrow}$ & 0.74 & $0.75^{\nearrow}$ & & \\
\hline & FIGR & 0.34 & $0.28^{\nearrow}$ & $0.22^{\nearrow}$ & $0.51^{\nearrow}$ & $0.43^{\nearrow}$ & $0.61^{\nearrow}$ & 0.8 & $0.72^{\nearrow}$ & $0.69^{\nearrow}$ & \\
\hline
\end{tabular}

" $\nearrow$ " indicates that the interaction type between the two driving factors is a nonlinear enhancement. The remaining interaction type is a bienhancement.

“*” denotes the largest interaction of each type (pairs of natural factors, pairs of socio-economic factors, and pairs of natural and socio-economic factors).

TABLE 5: Interaction of different factor pairs on urban expansion intensity during 2008-2015.

\begin{tabular}{|c|c|c|c|c|c|c|c|c|c|c|c|}
\hline \multirow{2}{*}{ Driving factors } & & \multicolumn{4}{|c|}{ Natural factors } & \multicolumn{6}{|c|}{ Socio-economic factors } \\
\hline & & DEM & Slope & DTR & DTC & $\mathrm{DRH}$ & PRG & GDPG & PCWG & FAIG & FIGR \\
\hline \multirow{4}{*}{ Natural factors } & DEM & & & & & & & & & & \\
\hline & Slope & 0.1 & & & & & & & & & \\
\hline & DTR & $0.13^{\nearrow}$ & $0.08^{\nearrow}$ & & & & & & & & \\
\hline & DTC & $0.23^{*}$ & 0.22 & 0.23 & & & & & & & \\
\hline \multirow{6}{*}{ Socio-economic factors } & DRH & 0.12 & 0.1 & $0.17^{\nearrow}$ & 0.28 & & & & & & \\
\hline & PRG & $0.16^{\nearrow}$ & $0.12^{\nearrow}$ & $0.23^{\nearrow}$ & $0.33^{\nearrow}$ & 0.13 & & & & & \\
\hline & GDPG & 0.32 & 0.3 & $0.42^{\nearrow}$ & 0.42 & $0.36^{\nearrow}$ & $0.60^{\nearrow}$ & & & & \\
\hline & PCWG & 0.21 & 0.18 & $0.33^{\nearrow}$ & $0.53^{\gamma_{*}}$ & 0.2 & $0.35^{\nearrow}$ & $0.79^{\nearrow *}$ & & & \\
\hline & FAIG & 0.3 & 0.28 & $0.35^{\nearrow}$ & 0.38 & $0.34^{\nearrow}$ & $0.61^{\nearrow}$ & $0.59^{\nearrow}$ & $0.71^{\nearrow}$ & & \\
\hline & FIGR & 0.16 & 0.12 & $0.20^{\nearrow}$ & $0.39^{\nearrow}$ & 0.12 & $0.24^{\nearrow}$ & $0.74^{\nearrow}$ & $0.31^{\nearrow}$ & $0.54^{\nearrow}$ & \\
\hline
\end{tabular}



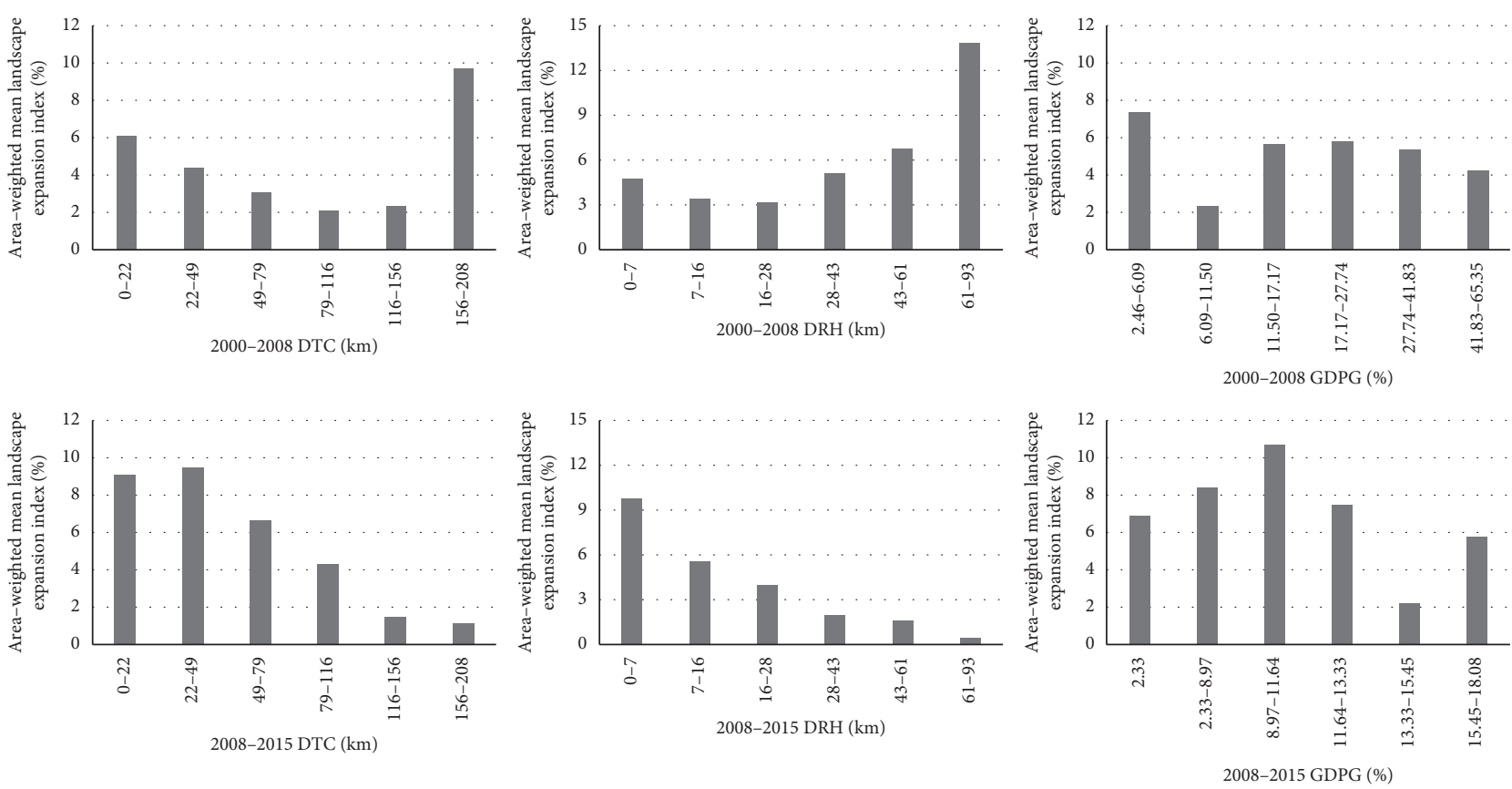

Figure 6: Risk analysis of DTC, DRH, and GDPG on urban expansion landscape pattern during 2000-2008 and 2008-2015.

investment in urban infrastructure construction in the previous period have greatly increased the demand for urban expansion. However, due to the implementation of policies on farmland resources and ecological environment protection by the national and local governments in the latter period, the UEI was greatly reduced, and the accumulation of labor in relatively developed coastal areas was mainly driven by the increase in per capita wages and living standards, which accelerated the process of population and land urbanization.

\subsection{Driving Mechanism of Urban Expansion Landscape Pattern}

5.2.1. Factor Analysis. According to the results of factor analysis, the driving effect of each factor on the AWMEI in PRD during 2008-2015 was much greater than that during 2000-2008. During 2000-2008, DTC, GDPG, DRH, and FIGR played a more important role on AWMEI than other factors. During 2008-2015, socio-economic factors drove AWMEI more than natural factors. Among socio-economic factors, the driving effects of PCWG, FAIG, and DRH were relatively large, while among natural factors, the driving effects of DTC and DEM were relatively large. The specific results were as follows: during 2000-2008, DTC (0.218) $>$ GDPG $\quad(0.160)>$ DRH $\quad(0.131)>$ FIGR $\quad(0.129)>$ PCWG $(0.056)>$ FAIG $(0.050)>$ PGR $(0.024)>$ DTR $(0.021)>$ DEM (0.008) > Slope (0.003); during 2008-2015, PCWG (0.672) $>$ FAIG $\quad(0.339)>$ DRH $\quad(0.259)>$ DTC $\quad(0.244)>$ GDPG $(0.214)>\operatorname{DEM}(0.170)>$ PGR $(0.152)>$ FIGR $(0.124)>$ Slope $(0.090)>$ DTR $(0.025)$. The following was a risk analysis of the factors that had relatively large driving effects on AWMEI in both periods, including DTC, DRH, and GDPG, thus revealing the average AWMEI of these driving factors in different risk areas.
5.2.2. Risk Analysis. Figure 6 showed that the further the distance to the coastline (DTC), the smaller the AWMEI. This was because PRD is dominated by an export-oriented economy. Regions near the coastline tended to be more densely populated, and urban land was well developed, making new urban land more interdependent with existing urban land. Similarly, the higher the GDPG, the smaller the AWMEI. The rapid economic development provided solid support for urban investment and construction and will undoubtedly accelerate the improvement of urbanization. However, the new urban land was more of an outlying expansion type in PRD during 2000-2015, which made the spatial pattern of urban land more loose. For DRH, the opposite laws were presented in the two periods: the farther away from the main road in the previous period, the greater the AWMEI; while the farther away from the main road in the latter period, the smaller the AWMEI. This indicated that the urban land in PRD has gradually expanded along the urban traffic. With the improvement of the transportation network system, the speed of the exchange of internal resources and information in the region greatly increased, which promoted the urban expansion. However, the newly added urban land was usually distributed sporadically along the main roads and continued to expand over time. Therefore, the spatial pattern of urban land became more compact in the latter period.

5.2.3. Interaction Analysis. The interaction between various factor pairs on AWMEI in 2008-2015 was significantly stronger than that in 2000-2008 (Tables 6 and 7). During 2000-2008, the average PD value of socio-economic factor pairs $(0.29)>$ natural and socio-economic factor pairs $(0.21)$ $>$ natural factor pairs (0.12); during 2008-2015, the average $\mathrm{PD}$ value of socio-economic factor pairs $(0.52)>$ natural and 
TABLE 6: Interaction of different factor pairs on urban expansion landscape pattern during 2000-2008.

\begin{tabular}{|c|c|c|c|c|c|c|c|c|c|c|c|}
\hline \multirow{2}{*}{ Driving factors } & & \multicolumn{4}{|c|}{ Natural factors } & \multicolumn{6}{|c|}{ Socio-economic factors } \\
\hline & & DEM & Slope & DTR & DTC & $\mathrm{DRH}$ & PRG & GDPG & PCWG & FAIG & FIGR \\
\hline \multirow{4}{*}{ Natural factors } & DEM & & & & & & & & & & \\
\hline & Slope & 0.01 & & & & & & & & & \\
\hline & DTR & $0.04^{\nearrow}$ & $0.03^{\nearrow}$ & & & & & & & & \\
\hline & DTC & $0.26^{\top}$ & $0.23^{\nearrow}$ & $0.36^{\gamma_{*}}$ & & & & & & & \\
\hline \multirow{6}{*}{ Socio-economic factors } & DRH & $0.16^{\nearrow}$ & $0.14^{\nearrow}$ & $0.17^{\nearrow}$ & $0.38^{\nearrow}$ & & & & & & \\
\hline & PRG & $0.04^{\nearrow}$ & $0.03^{\nearrow}$ & $0.12^{\nearrow}$ & $0.63^{\nearrow}$ & $0.18^{\nearrow}$ & & & & & \\
\hline & GDPG & $0.19^{\nearrow}$ & $0.17^{\nearrow}$ & $0.25^{\nearrow}$ & $0.69^{\gamma_{*} *}$ & $0.35^{\nearrow}$ & $0.35^{\nearrow}$ & & & & \\
\hline & PCWG & $0.09^{\nearrow}$ & $0.08^{\nearrow}$ & $0.11^{\nearrow}$ & $0.30^{\nearrow}$ & $0.39^{\nearrow}$ & $0.24^{\nearrow}$ & $0.55^{\nearrow}$ & & & \\
\hline & FAIG & $0.07^{\nearrow}$ & $0.06^{\nearrow}$ & $0.14^{\nearrow}$ & 0.25 & $0.26^{\nearrow}$ & $0.18^{\nearrow}$ & $0.41^{\nearrow}$ & $0.32^{\nearrow}$ & & \\
\hline & FIGR & $0.17^{\nearrow}$ & $0.15^{\nearrow}$ & $0.25^{\nearrow}$ & 0.33 & $0.35^{\nearrow}$ & $0.68^{\nearrow}$ & $0.69^{\gamma *}$ & $0.23^{\nearrow}$ & $0.26^{\nearrow}$ & \\
\hline
\end{tabular}

TABle 7: Interaction of different factor pairs on urban expansion landscape pattern during 2008-2015.

\begin{tabular}{|c|c|c|c|c|c|c|c|c|c|c|c|}
\hline \multirow{2}{*}{ Driving factors } & & \multicolumn{4}{|c|}{ Natural factors } & \multicolumn{6}{|c|}{ Socio-economic factors } \\
\hline & & DEM & Slope & DTR & DTC & $\mathrm{DRH}$ & PRG & GDPG & PCWG & FAIG & FIGR \\
\hline \multirow{4}{*}{ Natural factors } & DEM & & & & & & & & & & \\
\hline & Slope & 0.18 & & & & & & & & & \\
\hline & DTR & 0.19 & 0.11 & & & & & & & & \\
\hline & DTC & 0.3 & 0.28 & $0.30^{>*}$ & & & & & & & \\
\hline \multirow{6}{*}{ Socio-economic factors } & DRH & 0.3 & 0.29 & 0.27 & 0.37 & & & & & & \\
\hline & PRG & $0.34^{\nearrow}$ & 0.24 & $0.23^{\nearrow}$ & $0.50^{\nearrow}$ & 0.38 & & & & & \\
\hline & GDPG & 0.3 & 0.26 & $0.30^{\nearrow}$ & 0.42 & 0.36 & $0.65^{\nearrow}$ & & & & \\
\hline & PCWG & 0.71 & 0.69 & $0.71^{\nearrow}$ & $0.81^{*}$ & 0.73 & $0.83^{\nearrow}$ & $0.84^{*}$ & & & \\
\hline & FAIG & 0.43 & 0.38 & $0.43^{\nearrow}$ & 0.56 & 0.47 & $0.53^{\nearrow}$ & $0.62^{\nearrow}$ & 0.81 & & \\
\hline & FIGR & 0.27 & 0.2 & $0.22^{\nearrow}$ & $0.50^{\nearrow}$ & 0.32 & $0.58^{\nearrow}$ & $0.56^{\nearrow}$ & $0.82^{\nearrow}$ & $0.70^{\nearrow}$ & \\
\hline
\end{tabular}

socio-economic factor pairs $(0.41)>$ natural factor pairs (0.19). It can be found that AWMEI was always driven by socio-economic factor pairs more than natural factor pairs over 2000-2015, and the interaction of any two factors was an enhancement.

The interaction between DTR and DTC was the strongest among the natural factor pairs, whether in the previous period or the later period. This was mainly because PRD has been attracting foreign capital and technology by virtue of its superior coastal geographic location and preferential policies. The export-oriented economy was developing rapidly and the demand for urban land gradually expanded. At the same time, the dense water network in the region had a geographical impact on the landscape pattern of new urban land.

Among the socio-economic factor pairs, GDPG and FIGR had the strongest interaction during 2000-2008, and GDPG and PCWG had the strongest interaction during 2008-2015. This was because the economic growth of PRD and the increase of foreign direct investment in the previous period provided sufficient funds for regional industrial agglomeration, public facilities construction, which promoted the changes in landscape pattern of urban land. In the latter period, with the economic growth and the increase in per capita wages, regional living standards continued to increase, and the demand for urban housing, transportation, and infrastructure also increased, thus affecting the distribution of new urban land.
Among the natural and socio-economic factor pairs, DTC and GDPG had the strongest interaction during 2000-2008, and DTC and PCWG had the strongest interaction during 2008-2015. This was mainly due to the fact that PRD exerted its advantages of export-oriented economy, concentrated funds, information, and technology, and further promoted the optimization of industrial structure and urban land use pattern in the former period. The development of the export-oriented economy in the coastal areas and the increase in per capita wage resulted in a large concentration of labor in the latter period, expanding the demand for various types of urban land, thereby promoting the changes in the landscape pattern of the urban land.

\section{Discussion and Conclusion}

In general, the selection of driving factors that affect the urban expansion will vary according to the different study areas. According to the related research [15, 31, 38-40], this paper selected a total of 10 driving factors characterizing natural and socio-economic conditions related to urban expansion, and then quantitatively compare the spatiotemporal characteristics of driving mechanism. The results showed that natural factors were significantly weaker than socio-economic factors. This phenomenon could be explained by the short span of each study period, in which socio-economic factors tend to play a decisive role in urban expansion, which is confirmed in relevant studies [40, 41]. 
Thus, future research could expand the span of the study period to figure out how driving factors perform in different scenarios.

Besides, political factors also play an important role in urban expansion as mentioned before. However, they are difficult to be quantified when analyzing the driving mechanism of urban expansion. In this case, some quantitative factors can be used as the proxies to reflect the political factors, such as the socio-economic factors. Considering the impact of political factors on urban expansion, the interaction of natural, socio-economic, and political factors can be further explored.

Compared with the common quantitative methods, the method used in this study can better examine the interaction between different driving factors spatially and temporally. However, it also presents some limitations. First, the modifiable areal units of input geographical factors can yield different results. Therefore, the scale effect and the zoning effect of the input data should be considered [42]. Besides, the optimal scale effect and zoning effect might be different over space and time, which requires further exploration when analyzing the driving mechanism of urban expansion. Second, unlike the common quantitative methods, this method cannot predict or model future urbanization process based on the obtained results. This method can be further improved to make it more widely applicable on urban expansion.

In this study, the spatio-temporal characteristics of complex driving mechanism on urban expansion intensity and urban expansion landscape pattern were quantitatively explored. The main conclusions were as follows:

(1) The overall UEI of the PRD in 2000-2008 was higher than that in 2008-2015. Spatially, there was a dramatic and massive expansion of urban land around the estuary of the Pearl River in the previous period. While urban expansion was evenly distributed in the PRD without obvious expansion in the latter period. During 2000-2015, the most important land source for urban expansion was farmland, followed by forest land. In addition, outlying expansion was the main mode of urban expansion in the PRD, and the decline in its area proportion indicated that urban expansion has gradually become more compact.

(2) During the period 2000-2015, the socio-economic factors played a more important role on urban expansion intensity than the natural factors. Specifically, GDPG and FAIG were the two most important driving factors, but they showed different laws in the two periods. As for the natural factors, only DTC had a relatively large driving effect: the closer the distance to the coastline was, the higher the UEI was. Overall, the interaction between factor pairs on the UEI was significantly stronger in 2000-2008 than in 2008-2015. And both periods exhibited that socioeconomic factor pairs $>$ natural and socio-economic factor pairs $>$ natural factor pairs.

(3) Overall, the driving force of the driving factors on the urban expansion landscape pattern in 2008-2015 was far greater than that in 2000-2008. In the previous period, DTC, GDPG, DRH, and FIGR played a more important role than the other factors. While in the latter period, the socio-economic factors influenced the AWMEI more than the natural factors. In addition, the interaction between factor pairs on the AWMEI was significantly stronger in 2008-2015 than in 2000-2008. And both periods exhibited that socio-economic factor pairs $>$ natural and socioeconomic factor pairs $>$ natural factor pairs.

\section{Data Availability}

The data used to support the findings of the study are available from the corresponding author upon request.

\section{Conflicts of Interest}

The authors declare that they have no conflicts of interest.

\section{Acknowledgments}

This study was supported by the National Natural Science Foundation of China (41601082).

\section{References}

[1] A. Schneider and C. E. Woodcock, "Compact, dispersed, fragmented, extensive? A comparison of urban growth in twenty-five global cities using remotely sensed data, pattern metrics and census information," Urban Studies, vol. 45, no. 3 , pp. 659-692, 2008.

[2] S. Angel, J. Parent, D. L. Civco, A. Blei, and D. Potere, “The dimensions of global urban expansion: estimates and projections for all countries, 2000-2050," Progress in Planning, vol. 75, pp. 53-107, 2011.

[3] H. Zhou, M. Xu, R. Hou, Y. Zheng, Y. Chi, and Z. Ouyang, "Thermal acclimation of photosynthesis to experimental warming is season-dependent for winter wheat (Triticum aestivum L.)," Environmental and Experimental Botany, vol. 150, pp. 249-259, 2018.

[4] H. Xia, Y. Qin, G. Feng et al., "Forest phenology dynamics to climate change and topography in a geographic and climate transition zone: the qinling mountains in central China," Forests, vol. 10, no. 11, p. 1007, 2019.

[5] D. Lin, J. Xia, and S. Wan, "Climate warming and biomass accumulation of terrestrial plants: a meta-analysis," New Phytologist, vol. 188, no. 1, pp. 187-198, 2010.

[6] J. Xia and S. Wan, "Independent effects of warming and nitrogen addition on plant phenology in the Inner Mongolian steppe," Annals of Botany, vol. 111, no. 6, pp. 1207-1217, 2013.

[7] P. Silva and L. Li, "Mapping urban expansion and exploring its driving forces in the city of Praia, Cape Verde, from 1969 to 2015," Sustainability, vol. 9, no. 8, p. 1434, 2017.

[8] B. K. Terfa, N. Chen, D. Liu, X. Zhang, and D. Niyogi, "Urban expansion in Ethiopia from 1987 to 2017: characteristics, spatial patterns, and driving forces," Sustainability, vol. 11, no. 10, p. 2973, 2019.

[9] B. Song, S. Niu, and S. Wan, "Precipitation regulates plant gas exchange and its long-term response to climate change in a temperate grassland," Journal of Plant Ecology, vol. 9, no. 5, pp. 531-541, 2016. 
[10] X. Deng, J. Huang, S. Rozelle, and E. Uchida, "Growth, population and industrialization, and urban land expansion of China," Journal of Urban Economics, vol. 63, no. 1, pp. 96-115, 2008.

[11] J. Li, X. Deng, and K. C. Seto, "The impact of urban expansion on agricultural land use intensity in China," Land Use Policy, vol. 35, pp. 33-39, 2013.

[12] G. Tian, J. Jiang, Z. Yang, and Y. Zhang, "The urban growth, size distribution and spatio-temporal dynamic pattern of the Yangtze River Delta megalopolitan region, China," Ecological Modelling, vol. 222, no. 3, pp. 865-878, 2011.

[13] J. Liu, C. Han, J. Di, and H. Li, "Impact of climate-related changes to the timing of autumn foliage colouration on tourism in Japan," Tourism Management, vol. 70, pp. 262-272, 2019.

[14] M. Yan and J. Huang, "Review on the research of urban spatial expansion," Progress in Geography, vol. 32, pp. 1039-1050, 2013.

[15] Y. Ma and R. Xu, "Remote sensing monitoring and driving force analysis of urban expansion in Guangzhou City, China," Habitat International, vol. 34, no. 2, pp. 228-235, 2010.

[16] R. Camagni, M. C. Gibelli, and P. Rigamonti, "Urban mobility and urban form: the social and environmental costs of different patterns of urban expansion," Ecological Economics, vol. 40, no. 2, pp. 199-216, 2002.

[17] B. Shu, H. zhang, Y. Li, Y. Qu, and L. Chen, "Spatiotemporal variation analysis of driving forces of urban land spatial expansion using logistic regression: a case study of port towns in Taicang City, China," Habitat International, vol. 43, pp. 181-190, 2014.

[18] W. Kuang, Q. Shao, J. Liu, and C. Sun, "Spatio-temporal patterns and driving forces of urban expansion in Beijing central city since 1932," Geo-information Science, vol. 11, no. 4, pp. 428-435, 2010.

[19] G. Li, S. Sun, and C. Fang, "The varying driving forces of urban expansion in China: insights from a spatial-temporal analysis," Landscape and Urban Planning, vol. 174, pp. 63-77, 2018.

[20] S. S. Han, "Urban expansion in contemporary China: what can we learn from a small town?" Land Use Policy, vol. 27, no. 3, pp. 780-787, 2010.

[21] Y. Shi, J. Yang, and X. Hu, "How economic globalization affects urban expansion: an empirical analysis of 30 Chinese provinces for 2000-2010," Quality \& Quantity, vol. 50, no. 3, pp. 1117-1133, 2016.

[22] J. He, C. Li, J. Huang, D. Liu, and Y. Yu, "Modeling urban spatial expansion considering population migration interaction in Ezhou, central China," Journal of Urban Planning and Development, vol. 145, Article ID 05019003, 2019.

[23] Y. D. Wei, "Zone fever, project fever: development policy, economic transition, and urban expansion in China," Geographical Review, vol. 105, no. 2, pp. 156-177, 2015.

[24] Y. Long, Y. Gu, and H. Han, "Spatiotemporal heterogeneity of urban planning implementation effectiveness: evidence from five urban master plans of Beijing," Landscape and Urban Planning, vol. 108, no. 2-4, pp. 103-111, 2012.

[25] O. Dubovyk, R. Sliuzas, and J. Flacke, "Spatio-temporal modelling of informal settlement development in Sancaktepe district, Istanbul, Turkey," ISPRS Journal of Photogrammetry and Remote Sensing, vol. 66, no. 2, pp. 235-246, 2011.

[26] N. Haregeweyn, G. Fikadu, A. Tsunekawa, M. Tsubo, and D. T. Meshesha, "The dynamics of urban expansion and its impacts on land use/land cover change and small-scale farmers living near the urban fringe: a case study of Bahir Dar,
Ethiopia," Landscape and Urban Planning, vol. 106, no. 2, pp. 149-157, 2012.

[27] K. Müller, C. Steinmeier, and M. Küchler, "Urban growth along motorways in Switzerland," Landscape and Urban Planning, vol. 98, no. 1, pp. 3-12, 2010.

[28] M. Dong, P. Liu, Y. Zhang, and G. Yong, "Remote sensing monitoring and regional difference analysis of hetao oasis urbanization from 1975 to 2014," Bulletin of Soil and Water Conservation, vol. 4, pp. 1-8, 2016.

[29] R. B. Thapa and Y. Murayama, "Drivers of urban growth in the Kathmandu valley, Nepal: examining the efficacy of the analytic hierarchy process," Applied Geography, vol. 30, no. 1, pp. 70-83, 2010.

[30] K. C. Seto, M. Fragkias, B. Güneralp, and M. K. Reilly, "A meta-analysis of global urban land expansion,” PLoS One, vol. 6, 2011.

[31] S. Fang, G. Z. Gertner, Z. Sun, and A. A. Anderson, "The impact of interactions in spatial simulation of the dynamics of urban sprawl," Landscape and Urban Planning, vol. 73, no. 4, pp. 294-306, 2005.

[32] J. Li and L. Xie, "An analysis of the sustainable development of urbanization in the Pearl River Delta," Economic Geography, vol. 31, pp. 242-246, 2011.

[33] J. Liu, W. Kuang, Z. Zhang et al., "Spatiotemporal characteristics, patterns, and causes of land-use changes in China since the late 1980s," Journal of Geographical Sciences, vol. 24, no. 2, pp. 195-210, 2014.

[34] J. Y. Liu, M. L. Liu, D. F. Zhuang, Z. X. Zhang, and X. Z. Deng, "Study on spatial pattern of land-use change in China during 1995-2000," Science in China Series D-Earth Sciences, vol. 64, p. 373, 2003.

[35] J. Liu, W. Liu, W. Kuang, and J. Ning, "Remote sensing-based analysis of the spatiotemporal characteristics of built-up area across China based on the plan for major function-oriented zones," Acta Geographica Sinica, vol. 71, pp. 355-369, 2016.

[36] X. Liu, X. Li, Y. Chen, Z. Tan, S. Li, and B. Ai, "A new landscape index for quantifying urban expansion using multitemporal remotely sensed data," Landscape Ecology, vol. 25, no. 5, pp. 671-682, 2010.

[37] J. F. Wang, X. H. Li, G. Christakos et al., "Geographical detectors-based health risk assessment and its application in the neural tube defects study of the heshun region, China," International Journal of Geographical Information Science, vol. 24, no. 1, pp. 107-127, 2010.

[38] A. K. Braimoh and T. Onishi, "Spatial determinants of urban land use change in Lagos, Nigeria," Land Use Policy, vol. 24, 2007.

[39] A. M. Dewan and Y. Yamaguchi, "Land use and land cover change in Greater Dhaka, Bangladesh: using remote sensing to promote sustainable urbanization," Applied Geography, vol. 29, no. 3, pp. 390-401, 2009.

[40] T. Liu, G. Cao, J. Wang, B. S. Cui, and L. Yuan, "Progress in urban land expansion and its driving forces," Progress in Geography, vol. 29, pp. 927-934, 2010.

[41] L. Dai, H. Jiao, and J. Li, "Characteristics and influencing factors of urban spatial expansion in Wuhu," Journal of Anhui Normal University (Natural Science), vol. 35, pp. 371-375, 2012.

[42] D. E. Jelinski and J. Wu, "The modifiable areal unit problem and implications for landscape ecology," Landscape Ecology, vol. 11, no. 3, pp. 129-140, 1996. 